\title{
Jet and missing transverse momentum reconstruction in ATLAS at the LHC
}

\author{
Peter $\operatorname{Loch}^{a, b, *}$, on behalf of the ATLAS Collaboration \\ ${ }^{a}$ Department of Physics, University of Arizona, Tucson, Arizona 85721, USA \\ ${ }^{b}$ CERN, Geneva, Switzerland \\ E-mail: Peter.Loch@cern.ch
}

\begin{abstract}
Particle jets and the missing transverse momentum $\left(E_{\mathrm{T}}^{\text {miss }}\right)$ generated by the proton-proton collisions at the Large Hadron Collider (LHC) at CERN are important components of the final state reconstruction with the ATLAS detector. Both jet and $E_{\mathrm{T}}^{\text {miss }}$ reconstruction during LHC Run 2 (2015-2018), where the protons were collided with a centre-of-mass energy of $\sqrt{s}=13 \mathrm{TeV}$, faced significant challenges due to the high beam intensities that introduced the highest level of pile-up so far. Selected results from the evaluation of the jet and $E_{\mathrm{T}}^{\text {miss }}$ reconstruction performances are presented. Notably, the achieved precision of the jet energy measurement is about $1 \%$ over a large fraction of the accessible phase space for both narrow and wide jets, thus preserving the precision reached in previous LHC operations with significantly lower pile-up.
\end{abstract}

The Eighth Annual Conference on Large Hadron Collider Physics-LHCP2020

25-30 May, 2020

online

${ }^{*}$ Speaker 


\section{Introduction}

The hadronic final state of the proton-proton ( $p p$ ) collisions at the Large Hadron Collider (LHC) is reconstructed using particle jets representing hard parton emissions, the missing transverse momentum $\left(E_{\mathrm{T}}^{\text {miss }}\right)$ providing a proxy for the transverse momentum $\left(p_{\mathrm{T}}\right)$ of non-interacting particles, and several event shape variables measuring complex aspects of the overall $p_{\mathrm{T}}$ flow. During LHC Run 2, both jet and $E_{\mathrm{T}}^{\text {miss }}$ reconstruction in the ATLAS experiment [1] were significantly challenged due to the increased pile-up generated by up to 60 additional $p p$ interactions on average in the same bunch crossing as the signal event as well as by those in previous and following bunch crossings. This note summarizes the status of the jet and $E_{\mathrm{T}}^{\text {miss }}$ reconstruction efforts in ATLAS.

\section{Jet reconstruction}

ATLAS routinely uses two jet definitions in measurements and searches. Both employ the anti- $k_{t}$ jet algorithm [2] provided by the FastJet toolkit [3]. Narrow (small- $R$ ) jets are clustered with a distance parameter $R=0.4$ from particle flow (PFO) signals [4] combining reconstructed charged particle tracks emerging from the hard scatter vertex with calorimeter signals represented by clusters of topologically connected cell signals (topo-clusters) that are reconstructed at a basic (electromagnetic, EM) energy scale [5]. The principal reconstruction for small- $R$ jets following the jet clustering in both data and Monte Carlo (MC) simulations consists of (1) pile-up suppression using a jet-areabased approach [6] including a refinement for forward jets, (2) the jet energy scale (JES) calibration extracted from MC simulations and (3) a calibration refinement mitigating response variations due to jet flavour and energy leakage out of the calorimeter. Finally, jets in data only are calibrated to the MC JES using $p_{\mathrm{T}}$ balances in $\gamma / Z+$ jet and multi-jet final states, which are determined separately in-situ in data and MC simulations. A more detailed description of the small- $R$ jet reconstruction is given in Ref. [7].

Wide (large- $R$ ) jets are used as tools to e.g. tag boosted (heavy) particle decays where the decay products are collected into one jet. They are clustered with the anti- $k_{t}$ algorithm and $R=1$ from topo-clusters at the locally calibrated (LCW) energy scale [5]. Due to their large catchment area in rapidity-azimuth $(y, \phi)$ space and the observed variations of the $p_{\mathrm{T}}$ density from pile-up as a function of $y$, the jet-area-based method is found to be inefficient. The large- $R$ jets are trimmed instead, with a subjet clustering radius of $R=0.2$ and subjets dropped if their $p_{\mathrm{T}}$ is less than $5 \%$ of the original jet $p_{\mathrm{T}}$ [8]. As for the small- $R$ jets, the in-situ calibration for large- $R$ jets in data follows the MC-based JES calibration. The single jet mass is an important observable for e.g. jet origin tagging and searches for boosted decays. Therefore, the jet mass scale (JMS) is calibrated in addition to the JES in both data and MC simulations using functions derived from MC calibrations. To determine systematic uncertainties of the JMS, the jet mass spectra of hadronically decaying $W$ bosons and top quarks in MC simulations are forward-folded onto the corresponding distributions in data. The derived mass response and width adjustments then define the JMS uncertainties [9].

Figures 1(a), (b) show the data-to-MC simulation JES ratio from the various in-situ $p_{\mathrm{T}}$ balance measurements for small- $R$ and large- $R$ jets reconstructed in the central region of ATLAS. The combination of those measurements yields the calibration function for jets in data. The corresponding systematic uncertainties are shown in Figures 1(c), (d), respectively. In regions where in-situ 


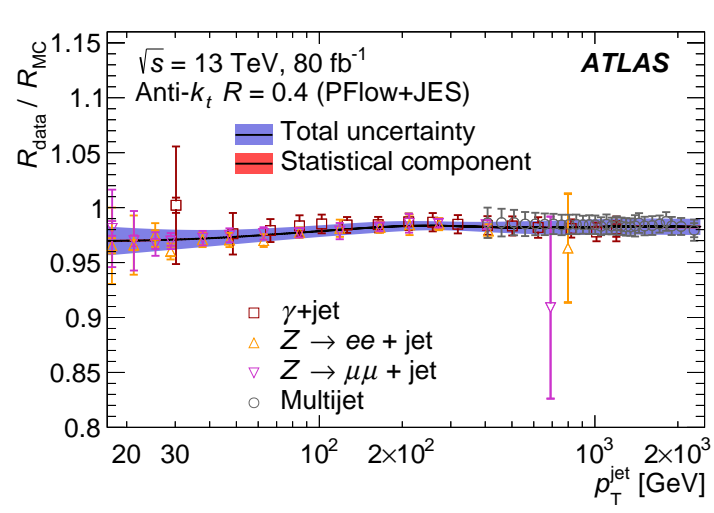

(a)

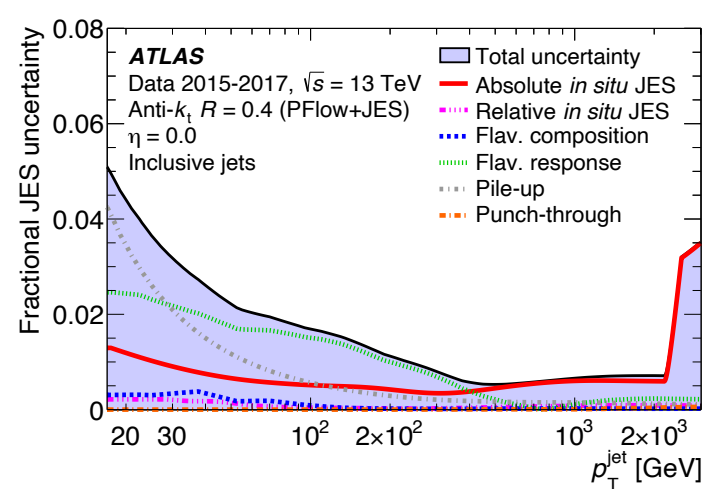

(c)

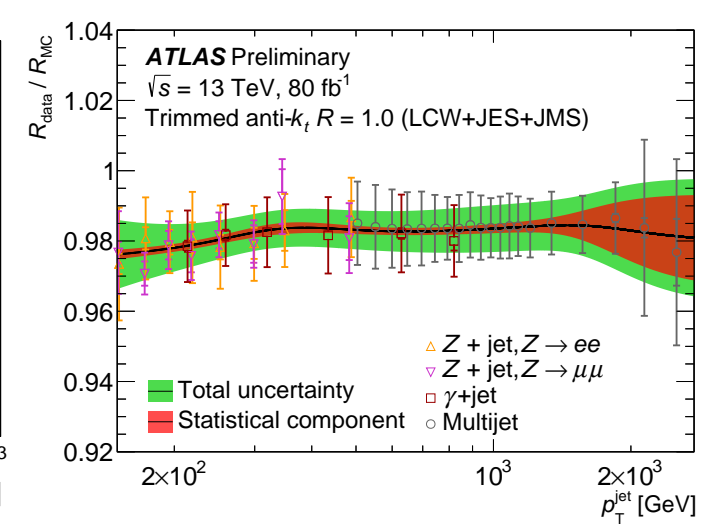

(b)

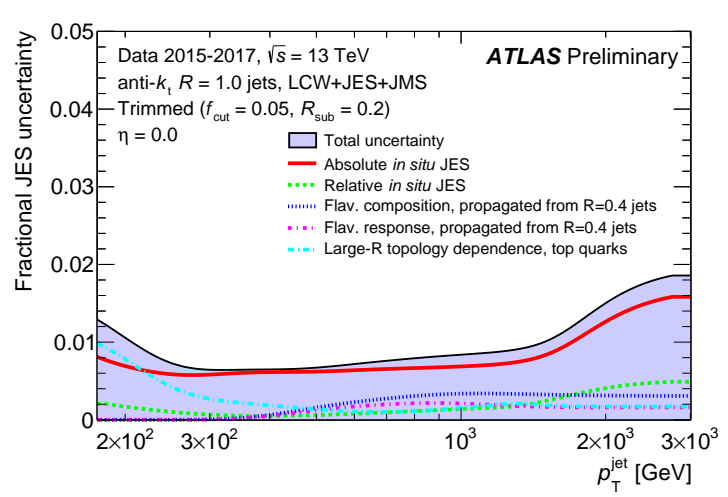

(d)

Figure 1: The ratios of the jet $p_{\mathrm{T}}$ responses in data and MC simulations for small- $R$ jets (a) from Ref. [7] and large- $R$ jets (b) from Ref. [10], as function of the jet- $p_{\mathrm{T}}$. Figure (c) from Ref. [7] shows the small- $R$ jet JES uncertainties for data, while (d) from Ref. [10] shows the JES uncertainties for large- $R$ jets in data.

calibration uncertainties from $\gamma /(Z \rightarrow \ell \ell)+$ jet final states dominate the overall JES uncertainty $\left(300 \mathrm{GeV} \lesssim p_{\mathrm{T}}^{\text {jet }} \lesssim 2 \mathrm{TeV}\right.$ ), a precision of $1 \%$ or better is observed for small- $R$ jets. The JES uncertainty for small- $R$ jets with $40 \mathrm{GeV} \lesssim p_{\mathrm{T}}^{\text {jet }} \lesssim 300 \mathrm{GeV}$ is dominated by uncertainties related to the jet flavour response, while the uncertainty in the $20 \mathrm{GeV}<p_{\mathrm{T}}^{\text {jet }} \lesssim 40 \mathrm{GeV}$ region is dominated by the uncertainty of the applied pile-up mitigation. The systematic JES uncertainties for large- $R$ jets are found to be less than $1 \%$, except for $p_{\mathrm{T}}^{\text {jet }} \lesssim 300 \mathrm{GeV}$ where final state topology dependencies of the response dominate, and for $p_{\mathrm{T}}^{\text {jet }} \gtrsim 2 \mathrm{TeV}$ where in-situ multijet balance is used. As for small- $R$ jets, the precision in this highest $p_{\mathrm{T}}^{\text {jet }}$ domain suffers from increased uncertainties of the reference $p_{\mathrm{T}}$ which reflect the propagation of JES uncertainties of lower- $p_{\mathrm{T}}$ jets.

\section{Missing transverse momentum reconstruction}

The $E_{\mathrm{T}}^{\text {miss }}$ reconstruction in ATLAS in LHC Run 2 follows an object-based approach [11]. Reconstructed final state particles and jets contribute to $E_{\mathrm{T}}^{\text {miss }}$ with priorities defined by the precision of their measurements and other quality requirements including isolation criteria, in addition to 


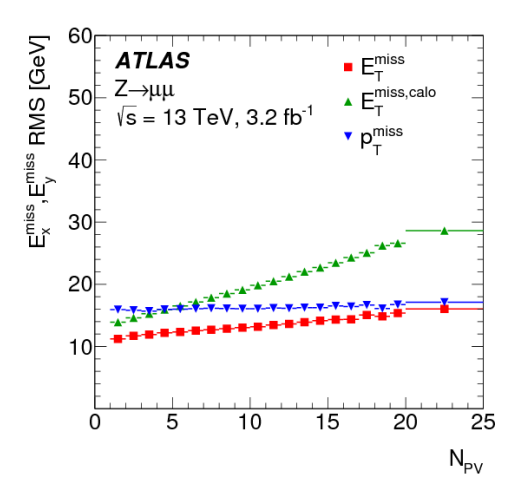

(a)

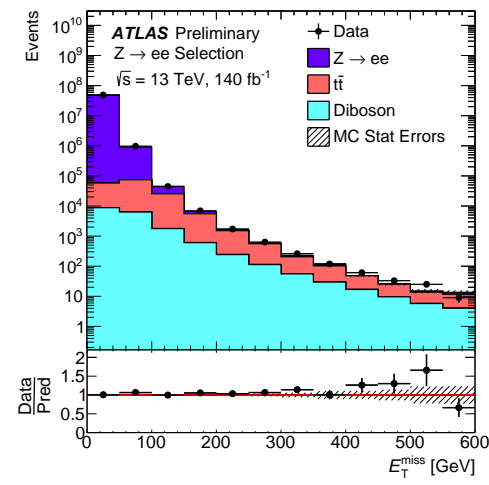

(b)

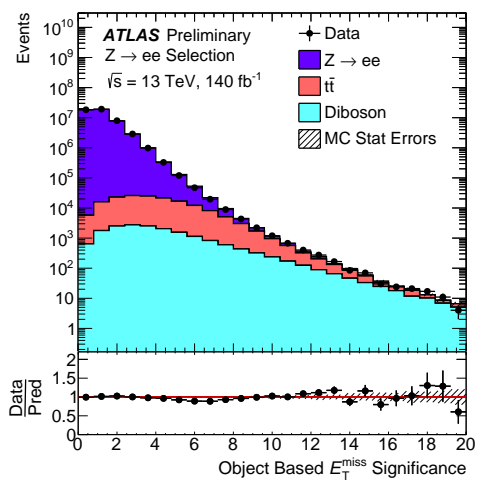

(c)

Figure 2: The $E_{\mathrm{T}}^{\text {miss }}$ resolution for an inclusive $Z \rightarrow \mu \mu$ data sample is shown as function of the pile-up measured by the number of reconstructed primary vertices $N_{\mathrm{PV}}$ in (a) from Ref. [11]. The overall $E_{\mathrm{T}}^{\text {miss }}$ spectrum from the same sample is shown for data and MC simulations in (b), while (c) compares the $E_{\mathrm{T}}^{\text {miss }}$ significance (both from Ref. [12]).

phase space restrictions, all possibly imposed in the context of a given analysis. If lower priority objects share signals with higher priority objects, they are typically ignored for $E_{\mathrm{T}}^{\text {miss }}$ to avoid double-counting. Reconstructed charged particle tracks not used in the reconstruction of the accepted objects and not overlapping or being otherwise associated with those contribute to $E_{\mathrm{T}}^{\text {miss }}$ as a soft term if they emerge from the hard scatter vertex, thus suppressing other possible soft signal contributions from pile-up.

This is illustrated in Figure 2(a) for an inclusive $Z \rightarrow \mu \mu$ sample in data, where the $E_{\mathrm{T}}^{\text {miss }}$ resolution as function of the pile-up activity is shown for three different reconstruction variants, (1) the standard reconstruction $\left(E_{\mathrm{T}}^{\text {miss }}\right)$, (2) the reconstruction with a soft term from calorimeter signals $\left(E_{\mathrm{T}}^{\text {miss,calo }}\right)$ and (3) a reconstruction of the whole missing transverse momentum using hard scatter vertex tracks only $\left(p_{\mathrm{T}}^{\text {miss }}\right)$. While $p_{\mathrm{T}}^{\text {miss }}$ shows no effect from pile-up, it does not provide the best resolution due to additional fluctuations in the recoil to the $Z$-boson $p_{\mathrm{T}}$ introduced by only considering the charged component. $E_{\mathrm{T}}^{\text {miss,calo }}$ is most affected by pile-up, as there is no solid indication of the signal origin (hard scatter or pile-up vertex) for the calorimeter signal. The good data-to-MC simulations agreement for $E_{\mathrm{T}}^{\text {miss }}$ and the associated $E_{\mathrm{T}}^{\text {miss }}$ significance [13] are shown in Figures 2(b), (c), respectively.

\section{Conclusion}

The jet and $E_{\mathrm{T}}^{\text {miss }}$ reconstruction in ATLAS for LHC Run 2 successfully addresses challenges introduced by the increasing pile-up. Levels of precision achieved in previous LHC operations are maintained and even exceeded, in particular for large- $R$ jets, with systematic uncertainties of around $1 \%$ for the jet energy measurement for both small- $R$ and large- $R$ jets over a large region of phase space. The track-based reconstruction of the $E_{\mathrm{T}}^{\text {miss }}$ soft term significantly reduces the pile-up dependence of the $E_{\mathrm{T}}^{\text {miss }}$ resolution. In addition, the $E_{\mathrm{T}}^{\text {miss }}$ significance is well described by MC simulations. This observable thus provides a useful tool for physics analyses. 


\section{References}

[1] ATLAS Collaboration, The ATLAS Experiment at the CERN Large Hadron Collider, JINST 3 S08003 (2008)

[2] Cacciari, M., Salam, G.P. and Soyez, G., The anti- $k_{t}$ jet clustering algorithm, JHEP 04063 (2008) [hep-ph/0802.1189]

[3] Cacciari, M.,Salam, G.P. and Soyez, G., FastJet User Manual, Eur.Phys.J.C 721896 (2012) [hep-ph/1111.6097]

[4] ATLAS Collaboration, Jet reconstruction and performance using particle flow with the ATLAS Detector, Eur.Phys.J.C 77466 (2017) [hep-ex/1703.10485]

[5] ATLAS Collaboration, Topological cell clustering in the ATLAS calorimeters and its performance in LHC Run 1, Eur.Phys.J.C 77490 (2017) [hep-ex/1603.02934]

[6] Cacciari, M.,Salam, G.P. and Soyez, G., The Catchment Area of Jets, JHEP 04005 (2008) [hep-ph/0802.1188]

[7] ATLAS Collaboration, Jet energy scale and resolution measured in proton-proton collisions at $\sqrt{s}=13 \mathrm{TeV}$ with the ATLAS detector, hep-ex/2007. 02645 (2020)

[8] Krohn, D., Thaler, J. and Wang, L.-T., Jet Trimming, JHEP 02084 (2010) [hep-ph/0912.1342]

[9] ATLAS Collaboration, In situ calibration of large-radius jet energy and mass in 13 $\mathrm{TeV}$ proton-proton collisions with the ATLAS detector, Eur.Phys.J.C 79135 (2019) [hep-ex/1807.09477]

[10] ATLAS Collaboration, ATLAS JETM-2019-05 https://atlas.web.cern.ch/Atlas/ GROUPS/PHYSICS/PLOTS/JETM-2019-05 (2019)

[11] ATLAS Collaboration, Performance of missing transverse momentum reconstruction with the ATLAS detector using proton-proton collisions at $\sqrt{s}=13 \mathrm{TeV}$, Eur.Phys.J.C 78903 (2018) [hep-ex/1802.08168]

[12] ATLAS Collaboration, ATLAS JETM-2019-03 https://atlas.web.cern.ch/Atlas/ GROUPS/PHYSICS/PLOTS/JETM-2019-03 (2019)

[13] ATLAS Collaboration, Object-based missing transverse momentum significance in the ATLAS detector, ATLAS-CONF-2018-038 http://cds . cern. ch/record/2630948 (2018) 